\title{
Accuracy of the Hodges-Lehmann estimates computed by applying Monte Carlo simulations
}

\author{
Robert Duchnowski $^{1}$ - Zbigniew Wiśniewski ${ }^{1}$
}

Received: 2 October 2015/Accepted: 16 September 2016/Published online: 28 September 2016

(C) The Author(s) 2016. This article is published with open access at Springerlink.com

\begin{abstract}
The paper concerns assessing the accuracy of some variants of robust R-estimates, namely the Hodges-Lehmann estimates, which can be applied, for example, in deformation analyses. Such estimates are robust against outlying observations and in some cases they are a good alternative for more conventional methods of estimation, for example, in testing stability of the potential reference points. Considering such an application, or in general estimation of displacements of network points, one should of course know accuracy of the estimators. Since R-estimates are based on ranks it is not obvious how to compute their accuracy (the law of variance propagation cannot be applied here). This paper presents one of the possible approaches, namely application of Monte Carlo simulations. If we make certain assumptions concerning the distribution of observation errors, we can assess the accuracy of chosen R-estimates. Usually, we assume that the observation errors are normally distributed, however, we can also consider some distributions with positive or negative kurtosis, and in the latter case we may apply the system of Johnson's distributions to simulate the observations. In the paper, the accuracy of R-estimates was computed in relation to the accuracy of LS estimates, which is advisable from a practical point of view. It turned out that the accuracy of R-estimates is a little bit worse than the accuracy of LS estimates in most of the cases. However, there are also some cases when R-estimates are more accurate, for example, for leptokurtic distributions of the observations. An example application of R-estimates in deformation analysis was also presented.
\end{abstract}

Keywords R-estimates · Robust estimates · Accuracy · Monte Carlo simulations

Robert Duchnowski

robert.duchnowski@uwm.edu.pl

1 Institute of Geodesy, University of Warmia and Mazury in Olsztyn, Olsztyn, Poland 


\section{Introduction and motivation}

Robust methods of estimation are well known and applied in several geodetic or surveying problems. Mostly they are used to deal with negative influence of outlying observations regardless of their sources. It is also well known that such methods, which are most often applied in geodetic practice, belong to the class called M-estimation. Here, we mean the estimation methods since there are also methods based on statistical tests, which are generally known as "data cleaning" (e.g., Baarda 1968; Hekimoglu and Erenoglu 2007; Prószyński 2015), where the outliers are supposed to be detected and rejected from the observation sets. Another possible approach to the problem of outliers is application of estimates that are based on ranks (R-estimates). The class of R-estimates is one of the most basic classes of robust estimations and it is often applied in many fields of science or practice. Such estimates can be also used in some geodetic or surveying computations (see, for example, Kargoll 2005; Duchnowski 2008). Here, we consider basic variants of R-estimates which are called Hodges-Lehmann's estimates (HLE); however, one can also apply their modified variants which consider different accuracies of the observations. Such approach was presented in (Duchnowski 2013), and the R-estimates proposed were called Hodges-Lehmann's weighted estimates (HLWE). There are two main variants of HLWE (or HLE, respectively), namely R-estimates of the expected value and R-estimates of the shift between two sets of observations. The latter kind of R-estimates is especially useful in deformation analyses (see, Duchnowski 2010, 2013). In such a case, it is of course necessary to know the accuracy of the estimates to identify possible displacements of network or object points. Thus, this paper focuses on the mentioned kinds of R-estimates but especially on the way in which their accuracy can be assessed. Since HLEs are based on the median (HLWEs on the weighted median, respectively), then the conventional method, which is based on the law of propagation of variance, cannot be applied here. Thus, we will apply one of the possible approaches to that problem, namely Monte Carlo simulations (other possible ways of assessing the accuracy of median-based estimates can be found in, e.g., Maritz and Jarret 1978; Hettmansperger and McKean 2011). The method which is based on Monte Carlo simulations was already applied for assessing the accuracy of several estimates (e.g., Duchnowski 2013; Duchnowski and Wiśniewski 2014; Shi and Xu 2015) and it seems to be very useful and advisable especially in the case of HLWE (which is more complicated case because of the application of the weighted median).

Duchnowski (2013) proposed to assess the accuracy of HLWE in relation to the accuracy of the respective least squares estimates (LSE) which seems very advisable from the practical point of view. Note that the accuracy of LSE can be easy computed, and if one knows the relation between such accuracy and the accuracy of HLWE (or HLE), then it is also easy to assess and accuracy of R-estimates. The respective proportionality factor $c$, which describes the relation between standard deviations of HLWE and LSE, must be of course computed from Monte Carlo simulations. In the paper in question, it was shown that $c \approx 1.07$. However, such value was based on simulations which were carried out for one example 2D network and for one of the possible variants of R-estimates and under the assumption that observation errors are normally distributed. Hence, the main objective of the paper is to assess the proportionality factor $c$ for different variants of R-estimates as well as different distributions assumed for the observation errors. 


\section{Hodges-Lehmann estimates in deformation analyses}

The basic variants of rank-based estimates were proposed by Hodges and Lehmann (1963), hence the name of that kind of estimators, namely Hodges-Lehmann's estimates. First, let us assume that random variables $X_{j}(1 \leq j \leq m)$ and also $Y_{i}(1 \leq i \leq n)$ are independently and identically distributed with the respective continuous distributions $F(x)$ and $G(y)=F(x-\Delta)$. Now, we can consider two independent samples $x_{1}, x_{2}, x_{3}, \ldots, x_{m}$ and $y_{1}$, $y_{2}, y_{3}, \ldots, y_{n}$ which are sets of realizations of those variables. When the Wilcoxon test statistic is applied, then we can get the well-known form of HLE of the shift $\Delta$ (the twosample problem)

$$
\hat{\Delta}^{H L}=\operatorname{med}\left(y_{i}-x_{j}\right)
$$

or HLE of the expected value $E(X)=E\left(X_{i}\right)$ (the one-sample problem)

$$
\hat{E}^{H L}(X)=\operatorname{med}\left(\frac{x_{j}+x_{k}}{2}\right)
$$

where med is a median and $1 \leq k \leq m$. Duchnowski (2013) proposed more general assumptions concerning distributions of the random variables which better reflect properties of geodetic or surveying measurements. Summing them up, let each set $x_{1}, x_{2}, x_{3}, \ldots$, $x_{m}$ and $y_{1}, y_{2}, y_{3}, \ldots, y_{n}$ contain realizations of different random variables $X_{j}$ or $Y_{i}$ that are independent, symmetrically distributed with the common respective expectations, namely $E(X)=E\left(X_{i}\right)$ or $E(Y)=E\left(Y_{i}\right)$, but may differ in variances. Such assumptions required application of a new test statistic which was also proposed in the paper mentioned. Finally, the new following estimates were obtained

$$
\hat{\Delta}^{H L W}=\operatorname{medw}\left(y_{i}-x_{j}\right)
$$

or

$$
\hat{E}^{H L W}(X)=\operatorname{medw}\left(\frac{x_{j}+x_{k}}{2}\right)
$$

where $m e d w$ is a weighted median [the definition and the ways in which the weighted median can be computed can be found in, e.g., (Gurwitz 1990; Duchnowski 2013)]. All variants of R-estimates can be applied in geodetic or surveying computations. It concerns especially the estimates of the shift which can be regarded as natural estimates of point displacements. In such a case, one can use the measurements to compute the coordinates of network (or object) points in several different and independent ways. Considering a particular point and two (or more) measurement epochs, such computed coordinates may stand in for the samples $x_{1}, x_{2}, x_{3}, \ldots, x_{m}$ and $y_{1}, y_{2}, y_{3}, \ldots, y_{n}$. Then, the way in which a particular point coordinate is changing between the measurement epochs can be estimated directly from R-estimates of the shift, Eqs. (1) or (3), or by comparing R-estimates of the expected values of the coordinate in the epochs in question, Eqs. (2) or (4) (see, Duchnowski 2013). There is also another possible approach which is based on initial values of residuals and which can be applied in the case of free networks (see, Duchnowski 2010); however we will not apply it here. Note that all four variants of HodgesLehmann estimates presented here are robust against outliers; however, their robustness differs from each other especially in the case of small samples (Rousseeuw and Verboven 2002; Duchnowski 2011; Duchnowski and Wiśniewski 2014). In the case of deformation analyses, where the samples contains usually a few elements, more robust are R-estimates of the shift, hence they are also more important from the practical point of view (Duchnowski 2011, 2013). 


\section{Monte Carlo simulations}

As it was already mentioned, the law of propagation of variance cannot be used to compute the variance of HLWE (or HLE) directly from the variances of the observations applied. Thus, we will assess the accuracy of the estimates in question by applying Monte Carlo simulations. The general idea behind that method is to simulate the sample $x_{1}, x_{2}, x_{3}, \ldots, x_{m}$, and in the cases of two-sample problem also the sample $y_{1}, y_{2}, y_{3}, \ldots, y_{n}$, by applying the chosen distribution and compute the particular R-estimate from Eqs. (1, 2, 3 or 4). Repeating such experiment one can get $N$ values of the estimate of the expected value or the shift. Such a set can be the basis for computing the empirical standard deviation $(\hat{\sigma})$ of the particular estimate. Such standard deviation will be used to compute the proportionality factor $c$ which describes the relation (Duchnowski 2013)

$$
\hat{\sigma}_{R}=c \cdot \hat{\sigma}_{L S E}
$$

where $\hat{\sigma}_{R}$ and $\hat{\sigma}_{L S E}$ are empirical standard deviations of a particular R-estimate or the least squares estimate, respectively (the value of $\hat{\sigma}_{L S E}$ can easily be computed by applying the law of propagation of variance). Thus, we will present the values of the coefficient $c$ instead of raw $\hat{\sigma}_{R}$. That has two practical purposes. The first one was already mentioned, if we know values of $c$, we will get a simple way of assessing the accuracy of R-estimates in a particular case. The second purpose is that we will also get information about accuracy of R-estimates in relation to the most often applied LSEs; hence, we can easily compare such estimates.

To carry out the simulation proposed here, we should choose the distributions of the observations. Of course, the most natural choice is the normal distribution, which is well grounded from the practical as well as theoretical point of view. However, some authors suggest that certain sets of geodetic observations show some anomalies in relation to the normal distribution (see, for example, Wiśniewski 1996, 2014). This may concern the higher order moments, and especially kurtosis $\gamma_{2}$. The term kurtosis is understood here as in the paper (Tadikamalla 1980), namely $\gamma_{2}=\mu_{4} / \mu_{2}^{2}-3$ ( $\mu_{i}$ is the $i$ th central moment); hence, for example, the kurtosis for normal distributions is equal to zero. It is noteworthy that sometimes the kurtosis, as implied here, is referred to as the coefficient of excess (or the excess kurtosis). Therefore, during the simulations we will consider also some leptokurtic $\left(\gamma_{2}>0\right)$ or platykurtic $\left(\gamma_{2}<0\right)$ distributions besides the normal one. To simulate leptokurtic or platykurtic distributions we will apply the Johnson system of distributions, namely the families of distributions called $S_{B}$ and $S_{U}$. Such families are defined by the following transformations (see, e.g., Tadikamalla 1980; Reed III and Stark 1995)

$$
S_{B}: Y=\xi+\frac{\lambda}{1+\exp \left\{\frac{-(Z-\gamma)}{\delta}\right\}}
$$

and

$$
S_{U}: Y=\xi+\lambda \cdot \sinh \left(\frac{Z-\gamma}{\delta}\right)
$$

where $Z$ is $N(0,1) ; \xi$ and $\lambda$ are the location and the scale parameters, respectively; $\gamma$ and $\delta$ are the shape parameters which determine the kurtosis and the asymmetry coefficient $\gamma_{1}$. Here, we will not consider asymmetric distributions, hence always $\gamma_{1}=0$. Under such an 
assumption we can apply $S_{B}$ to simulate platykurtic distributions, and $S_{U}$ to leptokurtic ones.

During all simulations we take the number of simulation $N=50,000$. In the case of shift estimates, we assume that $n=m$, namely both simulated samples have the same number of elements, which is acceptable from the practical point of view, for example, when we consider deformation analyses. Additionally, without loss of generality we assume that $E(X)=0$ and $\Delta=0$. The accuracy of R-estimates will be compared to accuracy of respective LSEs, namely an arithmetic mean or a weighted arithmetic mean (estimation of the expected value), and the respective differences of arithmetic or weighted arithmetic means (in the case of the shift estimates).

\subsection{Accuracy of HLE}

Let us assume without loss of generality that the standard deviation of all observations (the variables $X_{j}$ and $Y_{i}$ ) is equal to 1 (actually, we also carried out some additional simulations for other values of the standard deviation of observation and the results were the same). Now, let us simulate the following cases: all observations have normal distribution $\left(\gamma_{2}=0\right)$, or some leptokurtic distributions $S_{U}\left(\gamma_{2}>0\right)$, or platykurtic ones $S_{B}\left(\gamma_{2}<0\right)$. The obtained values of the proportionality factor $c$ for HLE of the expected value $\left(\hat{E}^{H L}(X)\right)$ are presented in Table 1, and for HLE of the shift $\left(\hat{\Delta}^{H L}\right)$ in Table 2, respectively.

One should add that for $n=2$ HLEs are exactly the same as respective LSEs, thus $c=1$. The most important, at least from the practical point of view, are the results obtained for the normal distribution. In such a case, we can note that both HLEs are a little bit less accurate than respective LSEs, and what is more the value of $c$ is almost independent of $n$. It is also noteworthy that the more leptokurtic the distribution is, the more accurate HLEs are (in relation to LSEs). The only exception is the case of $n=3$ for $\hat{E}^{H L}(X)$, where one can observe the opposite effect. One should also remember that the distributions with the extreme values of kurtosis have less importance in practice.

\subsection{Accuracy of HLWE}

In the case of HLWEs, we assume that the variances of the variables $X_{j}$ (but also $Y_{i}$ ) may differ from each other, or in other words the accuracies of the observations may be different from each other. That should be reflected within the simulations. Since it is impossible to consider all possible cases, we propose the following scheme of the simulations. Consider a particular case of $n$ observations. Now, let us choose a certain $k$ such that $1 \leq k \leq n-1$, and let the first $k$ observations have the assumed standard deviations

Table 1 Values of the factor $c$ for HLE of the expected value

\begin{tabular}{llllllllll}
\hline$\gamma_{2}$ & \multicolumn{1}{l}{ Number of observations $n$} \\
\cline { 2 - 9 } & 3 & 4 & 5 & 6 & 7 & 8 & 9 & 10 & 20 \\
\hline-1 & 0.96 & 1.14 & 1.10 & 1.07 & 1.10 & 1.11 & 1.10 & 1.09 & 1.09 \\
-0.5 & 1.02 & 1.08 & 1.06 & 1.05 & 1.06 & 1.06 & 1.06 & 1.06 & 1.06 \\
0 & 1.04 & 1.05 & 1.03 & 1.03 & 1.04 & 1.03 & 1.03 & 1.03 & 1.03 \\
3 & 1.12 & 0.96 & 0.96 & 0.96 & 0.95 & 0.94 & 0.94 & 0.94 & 0.92 \\
7 & 1.15 & 0.91 & 0.91 & 0.91 & 0.90 & 0.89 & 0.89 & 0.88 & 0.85 \\
12 & 1.18 & 0.87 & 0.87 & 0.88 & 0.86 & 0.85 & 0.84 & 0.83 & 0.81 \\
\hline
\end{tabular}


Table 2 Values of the factor $c$ for HLE of the shift

\begin{tabular}{llllllllll}
\hline$\gamma_{2}$ & \multicolumn{1}{l}{ Number of observations $n=m$} \\
\cline { 2 - 9 } & 3 & 4 & 5 & 6 & 7 & 8 & 9 & 10 & 20 \\
\hline-1 & 1.08 & 1.10 & 1.10 & 1.10 & 1.10 & 1.10 & 1.09 & 1.09 & 1.08 \\
-0.5 & 1.07 & 1.07 & 1.08 & 1.07 & 1.07 & 1.07 & 1.07 & 1.07 & 1.06 \\
0 & 1.05 & 1.05 & 1.05 & 1.04 & 1.04 & 1.04 & 1.04 & 1.04 & 1.03 \\
3 & 0.99 & 0.96 & 0.96 & 0.95 & 0.95 & 0.94 & 0.93 & 0.93 & 0.92 \\
7 & 0.95 & 0.91 & 0.90 & 0.89 & 0.88 & 0.87 & 0.87 & 0.87 & 0.85 \\
12 & 0.92 & 0.87 & 0.87 & 0.84 & 0.84 & 0.83 & 0.83 & 0.82 & 0.80 \\
\hline
\end{tabular}

computed by the following formula $\sigma_{l}=1+\alpha \cdot l, 1 \leq l \leq k$, and $\alpha$ is an arbitrary but known value (such that for all $l \sigma_{l}>0$ ). Additionally, let us assume that the standard deviations of the rest of the observations, namely $n-k$ observations, are the same and equal to 1 . By taking different values of $l$ and $\alpha$ one can get many various cases. For example, if $n=4, k=2$ and $\alpha=1$ then the sets of observations are simulated under the assumption that $\sigma_{1}=2, \sigma_{2}=3, \sigma_{3}=1$ and $\sigma_{4}=1$. Tables 3 and 4 show the values of the proportionality factor $c$ for HLWE of the expected value $\left(\hat{E}^{H L W}(X)\right)$ and for HLWE of the shift $\left(\hat{\Delta}^{H L W}\right)$, respectively, and which were obtained for normal distributions of the observations.

The results obtained for $n=2$ need some explanations. In the case of HLWE of the expected value Eq. (4), it is easy to notice that when $n=2$, then $\hat{E}^{H L W}(X)$ is equal to the arithmetic mean of two observations (if only the standard deviations of observations do not differ much from each other). Thus for small $\alpha$, we get $c$ close to 1 . When the difference between standard deviations of the observations becomes significant, then the weighted

Table 3 Values of the factor $c$ for HLWE of the expected value

\begin{tabular}{|c|c|c|c|c|c|c|c|}
\hline \multirow[t]{2}{*}{$n$} & \multirow[t]{2}{*}{$k$} & \multicolumn{6}{|l|}{$\alpha$} \\
\hline & & 0.1 & 0.2 & 0.5 & 1 & 2 & 5 \\
\hline 2 & 1 & 1.00 & 1.02 & 1.08 & 1.25 & 1.06 & 1.01 \\
\hline \multirow[t]{2}{*}{3} & 1 & 1.05 & 1.05 & 1.03 & 1.06 & 1.03 & 1.01 \\
\hline & 2 & 1.06 & 1.05 & 1.08 & 1.10 & 1.07 & 1.02 \\
\hline \multirow[t]{3}{*}{4} & 1 & 1.05 & 1.05 & 1.06 & 1.06 & 1.04 & 1.04 \\
\hline & 2 & 1.06 & 1.06 & 1.05 & 1.05 & 1.04 & 1.01 \\
\hline & 3 & 1.06 & 1.05 & 1.07 & 1.10 & 1.09 & 1.02 \\
\hline \multirow[t]{4}{*}{5} & 1 & 1.04 & 1.04 & 1.04 & 1.04 & 1.06 & 1.06 \\
\hline & 2 & 1.04 & 1.04 & 1.05 & 1.05 & 1.05 & 1.05 \\
\hline & 3 & 1.04 & 1.05 & 1.06 & 1.05 & 1.04 & 1.01 \\
\hline & 4 & 1.05 & 1.05 & 1.08 & 1.10 & 1.09 & 1.02 \\
\hline \multirow[t]{2}{*}{6} & 1 & 1.03 & 1.03 & 1.04 & 1.04 & 1.04 & 1.04 \\
\hline & 5 & 1.04 & 1.05 & 1.08 & 1.11 & 1.09 & 1.02 \\
\hline \multirow[t]{2}{*}{7} & 1 & 1.04 & 1.03 & 1.04 & 1.03 & 1.03 & 1.03 \\
\hline & 6 & 1.04 & 1.05 & 1.08 & 1.12 & 1.10 & 1.02 \\
\hline \multirow[t]{2}{*}{10} & 1 & 1.03 & 1.03 & 1.04 & 1.03 & 1.03 & 1.03 \\
\hline & 9 & 1.04 & 1.06 & 1.09 & 1.12 & 1.10 & 1.02 \\
\hline
\end{tabular}


Table 4 Values of the factor $c$ for HLWE of the shift

\begin{tabular}{llllllll}
\hline$n=m$ & $k$ & $\alpha$ & & & & & \\
\cline { 3 - 7 } & & 0.1 & 0.2 & 0.5 & 1 & 2 & 5 \\
\hline 2 & 1 & 1.13 & 1.10 & 1.07 & 1.09 & 1.06 & 1.01 \\
3 & 1 & 1.06 & 1.07 & 1.06 & 1.08 & 1.09 & 1.12 \\
& 2 & 1.06 & 1.07 & 1.08 & 1.09 & 1.07 & 1.02 \\
4 & 1 & 1.06 & 1.06 & 1.06 & 1.07 & 1.06 & 1.06 \\
& 2 & 1.06 & 1.06 & 1.07 & 1.08 & 1.09 & 1.12 \\
& 3 & 1.06 & 1.07 & 1.09 & 1.09 & 1.08 & 1.02 \\
5 & 1 & 1.05 & 1.05 & 1.05 & 1.06 & 1.06 & 1.06 \\
& 2 & 1.05 & 1.06 & 1.06 & 1.06 & 1.06 & 1.06 \\
& 3 & 1.05 & 1.06 & 1.07 & 1.08 & 1.09 & 1.12 \\
& 4 & 1.05 & 1.06 & 1.08 & 1.10 & 1.08 & 1.02 \\
6 & 1 & 1.05 & 1.05 & 1.05 & 1.05 & 1.05 & 1.05 \\
& 5 & 1.05 & 1.06 & 1.08 & 1.09 & 1.08 & 1.02 \\
7 & 1 & 1.05 & 1.04 & 1.05 & 1.05 & 1.05 & 1.05 \\
& 6 & 1.05 & 1.06 & 1.08 & 1.10 & 1.08 & 1.02 \\
& 1 & 1.04 & 1.04 & 1.04 & 1.04 & 1.04 & 1.04 \\
& 9 & 1.05 & 1.06 & 1.09 & 1.10 & 1.08 & 1.02 \\
\hline \multirow{2}{*}{5} & & & & & & &
\end{tabular}

mean (here, LSE) is more accurate than the arithmetic mean, and hence more accurate than $\hat{E}^{H L W}(X)$. The value of $c$ grows. There is a particular value of $\alpha$ for which such an effect is maximum (in our simulations it is for $\alpha=1$ ). Later on, $\hat{E}^{H L W}(X)$ is no longer equal to the mean but its value depends strongly on the value of the observation with the bigger weight. Therefore, after reaching the maximum at $\alpha=1, c$ begins to decrease. A similar effect, but with a different magnitude, can also be observed for other values of $n$ and $k$.

The situation is a little bit more complicated for HLWE of the shift, Eq. (3). The values of $c$ obtained for the smaller $\alpha$ are amazingly big (see, Table 4 for $n=2$ ), especially if we remind, that for $n=m=2$ and $\alpha=0$ we should get $c=1$. That results from the general properties of the weighted median in the two-sample problem. To explain that effect, let us assume that $n=m=2$ and the respective variables $X_{j}$ and $Y_{j}$ have the same standard deviations. Then, considering Eq. (3), one should compute the weighted median of a sample which contains four elements. Note that that if $\alpha \neq 0$ then two of them have the same weights, the other two have two different weights; however, for small $\alpha$ all such weights will be close to each other. Thus, the weighted media will be equal to the second or the third value within the ordered sample, but never equal to the mean of those two values. From a statistic point of view, such value may be relatively far from the theoretical value $\Delta=0$. Therefore, the values of $c$ are bigger for small $\alpha$. Note that if one neglects the differences of the weights and assumes the same weight for all observations, then the weighted median will be equal to the mean of the second and third values and such estimate will be more accurate ( $c$ will be close to 1). On the other hand, the bigger is $\alpha$, the more varied are the weights. Thus, when $\alpha$ is growing, the value with the biggest weight will become more and more "important" within the sample (statistically such a value should be closest to the $\Delta=0$ ); hence, the smaller values of $c$ for bigger values of $\alpha$. This effect is characteristic only for $n=m=2$. As for the other cases, if $k=1$ and $n=m>3$, then $c$ is almost independent from $\alpha$. If $k=n-1$, then one can observe the effect which is 
similar to that described in the case of $\hat{E}^{H L W}(X)$. Firstly, $c$ grows up but when $\alpha$ exceeds 1 , it begins to decrease. For other values of $k, c$ usually grows up slightly with increasing $\alpha$.

Considering practical geodetic or surveying applications of HLWEs, for example in deformation analyses, we should pay special attention to the simulations with relatively small $\alpha$. The reason is that observations of the same type have usually similar accuracy (within a particular network). That also concerns application of HLWE in deformation analyses, when coordinates of a particular network point are computed for several times. The numerical example, which concerns 2D network and which is presented in (Duchnowski 2013), shows that standard deviations of such coordinates are usually similar and the maximum differences do not exceed $50 \%$. Thus, the results of the simulations for which $\alpha \leq 1$ are the most important here, and the simulations for $\alpha=2$ or 5 , have rather theoretical meaning.

Let us now examine the accuracy of HLWE when observations are not normally distributed. Within the simulations, we assume that all observations have the same expected value, the same kurtosis but may differ from one another in the standard deviations. Now, let us choose the following case $k=1, \alpha=1$, and let $c$ be computed for different $n$ and for some leptokurtic or platikurtic distributions of observations (by applying $S_{U}$ or $S_{B}$ distributions). The results of such simulations are presented in Figs. 1 and 2, respectively.

The results seem to be very similar for those obtained in the case of HLEs, the values of $c$ are smaller for leptokurtic distributions and bigger for the platikurtic ones. Note that if $n \geq 4$ and the distributions are leptokurtic then HLWEs are even better than respective LSEs. However, the results presented in Figs. 1 and 2 concern only one of the possible cases. Thus, let us also consider another variant, namely let $n=5, k=2$, and the values of $c$ be computed for different values of $\alpha$ (see, Figs. 3, 4). Figure 4, which presents results for HLWE of the shift, confirms the conclusions presented above. The situation is different for HLWE of the expected value (Fig. 3). If $\alpha$ is small, then the better result are obtained for the leptokurtic distributions but when $\alpha>2$ then one can observe the opposite effect. Thus, while applying such an estimate we cannot be certain that its accuracy is better for the leptokurtic or platikurtic distributions. On the other hand, we already mentioned that in surveying practice we can often assume that observations have similar accuracies (small $\alpha$ ), thus we can expect better accuracy for leptokurtic distributions in practical applications of HLWE of the expected value.

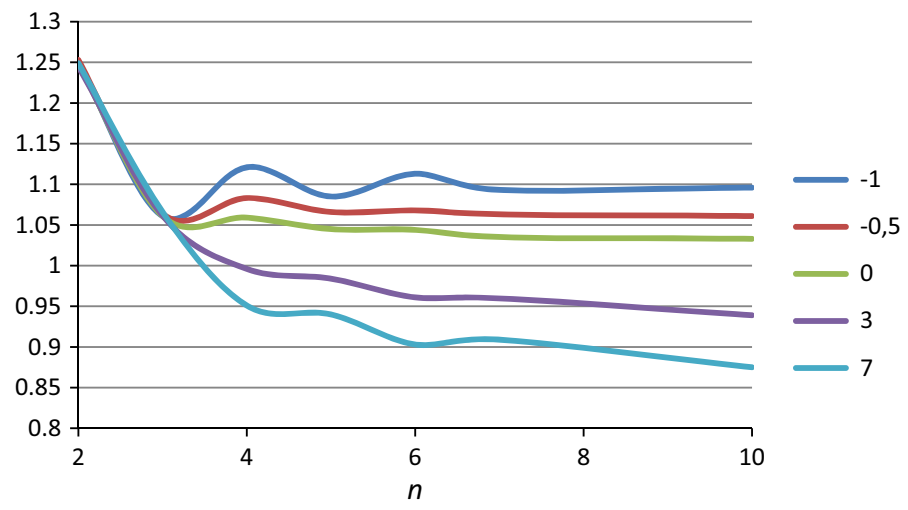

Fig. 1 Values of the factor $c$ for HLWE of the expected value for $k=1, \alpha=1$ and the distributions with different kurtosis $\gamma_{2}$ 


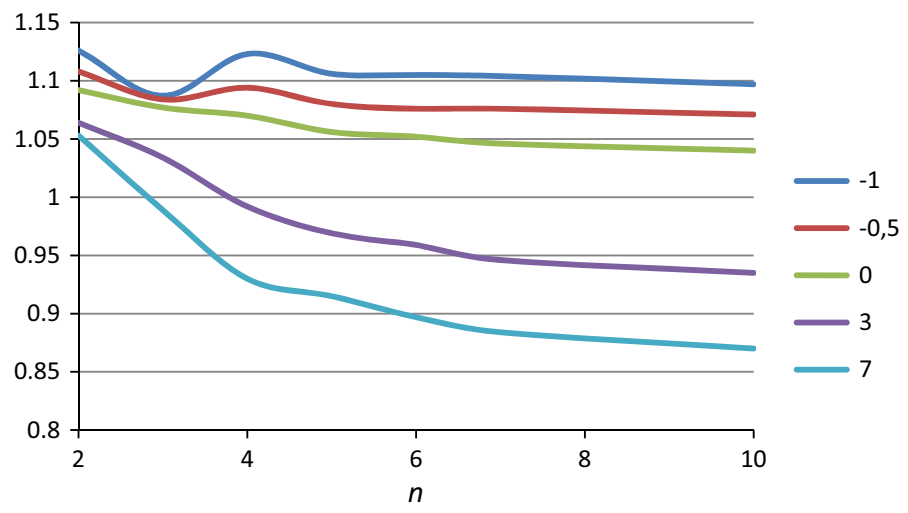

Fig. 2 Values of the factor $c$ for HLWE of the shift for $k=1, \alpha=1$ and the distributions with different kurtosis $\gamma_{2}$

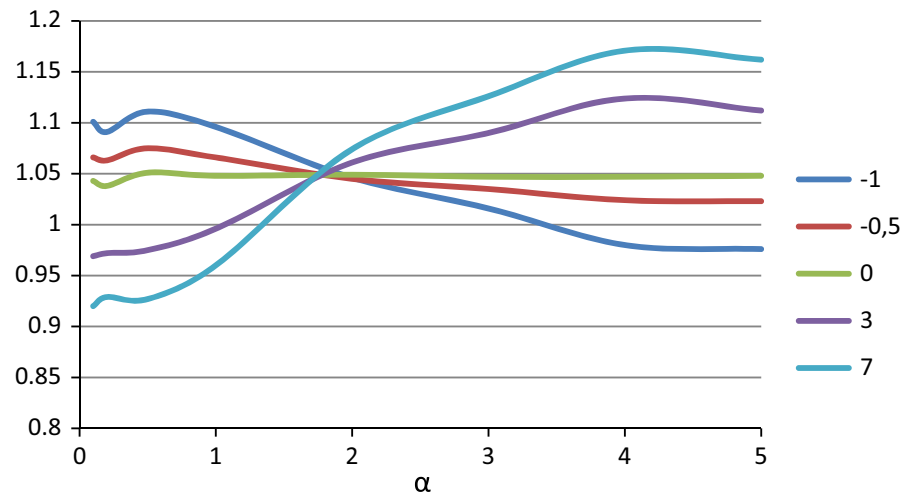

Fig. 3 Values of the factor $c$ for HLWE of the expected value for $n=5, k=2$ and the distributions with different kurtosis $\gamma_{2}$

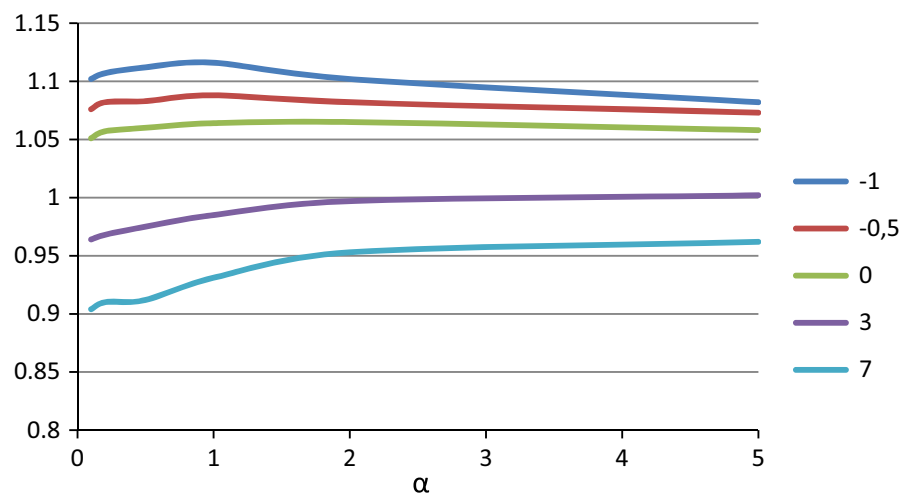

Fig. 4 Values of the factor $c$ for HLWE of the shift for $n=5, k=2$ and the distributions with different kurtosis $\gamma_{2}$ 


\section{Example application}

Now let us present an application of R-estimates in deformation analysis. Consider a network which was designed for the dam Montsalvens in Switzerland, which was built in 1921. The main network purpose was to monitor deformation of the dam. We will analyze a part of that network, which is presented in Fig. 5. Thus, we will check the stability of five potential reference points. Such a network was already considered as an example in some previous publications (see, Caspary 2000; Nowel 2015), which gives us possibility to compare the final results of different approaches to deformation analysis. The detailed description of the network can be found in the mentioned papers, here let us summarize that we consider two measurement epochs, and the two observation sets consist of five arcs of horizontal directions with the assumed standard deviation $\sigma_{a}=3^{c c}$ and six distances with $\sigma_{d}=0.3[\mathrm{~mm}]$. We consider only a part of the network; however, the a priori standard deviations and the network scale are realistic. We apply the local coordinate system which is advisable for such purposes. All the observations applied in the computations as well as other required data can be found in (Caspary 2000). In accordance with that paper, the observations are simulated for two independent epochs and under the assumption that Point 3 is unstable (with the assumed horizontal displacements of $\delta x=-0.50[\mathrm{~mm}], \delta y=-0.60[\mathrm{~mm}])$.

HLEs (but also HLWEs) are natural estimates of the shift between two samples, thus, they can also be applied to test stability of the potential reference points, PRPs (see, Duchnowski 2010, 2013). To illustrate such an application let us consider only a part of the network in question and let us test stability of PRPs by applying HLWEs and their accuracies estimated by the method presented in this paper. The main idea behind application of HLWEs in this context is to compute the coordinates of the chosen point in several independent ways (by applying the initial values of the coordinates of the other points, the known observations and simple surveying methods like, for example, angular or linear intersections, resection etc.). Given such coordinate sets for both measurement epochs, one can apply HLWE to estimate the shift between those sets, which in fact is equal to the estimated change of the point coordinate (X or $\mathrm{Y}$ ). Now, let us present the way

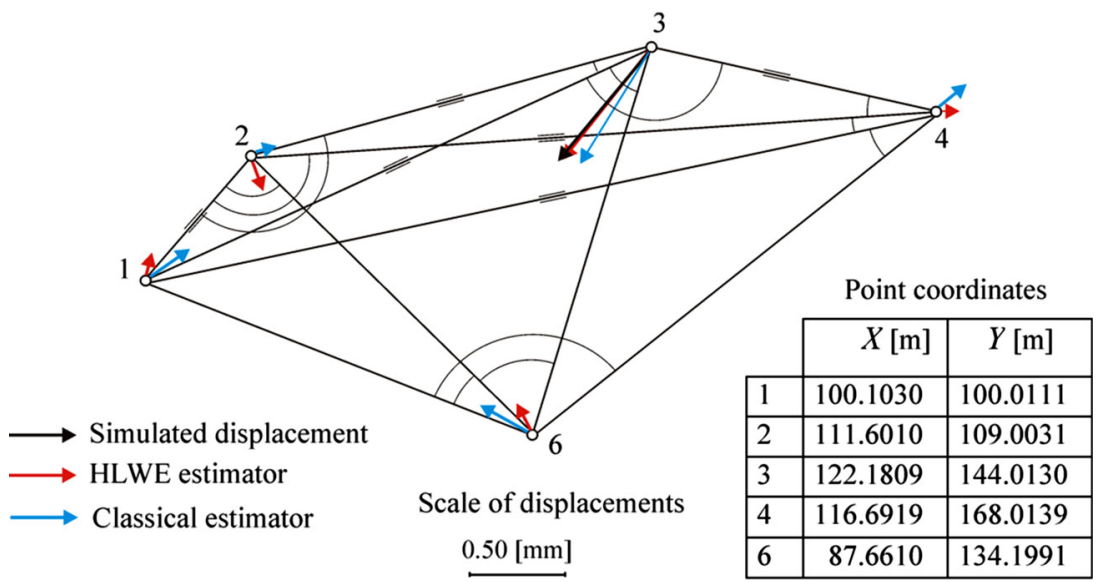

Fig. 5 Part of the dam monitoring network Montsalvens with displacement of the network point (simulated and estimated) 
of the computation in the case of the point 1 and the coordinate X1. First, we should assume the initial values of the coordinates of all network points. Cymerman et al. (2016) discussed such a problem for the levelling networks and showed that one can apply the initial values computed on the basis of the first epoch observations, namely the approximate coordinates or the adjusted coordinates. In the latter approach we should of course neglect the correlation between such computed coordinated, which is admissible (Cymerman et al. 2016). Here, we will apply the adjusted coordinates from the first epoch (Caspary 2000); however, we also performed the computation in the case of the approximate coordinates, and generally the results were the same. The second necessary assumption in the case of the particular network concerns the horizontal angles. Since they are computed by applying the measured horizontal directions, they might be correlated. In the case of HLWEs we should neglect such a correlation (note that such an assumption would be justified if not the directions themselves but the appropriate angles were measured independently, which is possible and plausible in the practice).

Under such assumptions and considering the network configuration one can compute the coordinate $\mathrm{X} 1$ in 8 independent ways, namely by 6 angular intersections, 1 linear intersection and one reception (see, Fig. 5). Such computed coordinates and their accuracies are presented in Table 5. Of course the same computations can be repeated for the second epoch (note that the same initial coordinates are also assumed for the second epoch). Such sets of the coordinates are the basis for computing HLWE of the change of the coordinate $\mathrm{X} 1$ (see, Eq. 3). The similar computations can be performed for $\mathrm{Y} 1$ as well as for the coordinates of the rest of PRPs. In the case of the points 2, 3, 4 one can apply: 6 angular intersections, 1 linear intersection and one reception; and for the point 6 there are 6 independent angular intersections and 1 resection. The next step is to assess the accuracy of the estimates obtained. This can be performed by applying new Monte Carlo simulations or in the simplified way, namely with application the factors $c$, which were already computed and which were listed in Table 4. The estimation results are presented in Table 6, which also presents the results obtained by applying the classical method (Caspary 2000) or the method which is based on robust M-estimation (Nowel 2015).

Now let us find stable reference frame within PRPs. Duchnowski (2011) showed that unstable points are the source of outlying observations when stability of other PRPs is tested by applying HLEs or HLWEs. In our case, the unstable point 3 disturbs the testing of stability for the points 1, 2, 4 and 6. Namely, the coordinates of those four points computed by applying the observations which contain the point 3 might be regarded as outlying (at the second epoch). For example, in the case of the point 1 , there are 8 computed values of the coordinate X1, 4 of which might be outliers (see, Table 5). Note that such outliers do

Table 5 Values of the coordinate $\mathrm{X} 1$ computed by applying six angular intersections, one linear intersection and one resection

*Angles: left-central-right, distances: $i-j$

\begin{tabular}{lll}
\hline Observations applied ${ }^{*}$ & Coordinate X1 $(\mathrm{m})$ & $\sigma_{X 1}(\mathrm{~mm})$ \\
\hline $1-6-2,6-2-1$ & 100.10322 & 0.19 \\
$1-6-3,6-3-1$ & 100.10317 & 0.30 \\
$1-6-4,6-4-1$ & 100.10322 & 0.91 \\
$1-4-2,4-2-1$ & 100.10283 & 0.50 \\
$1-4-3,4-3-1$ & 100.10165 & 1.90 \\
$1-3-2,3-2-1$ & 100.10200 & 0.24 \\
$4-1,3-1$ & 100.10328 & 1.77 \\
$2-1-4,4-1-6$ & 100.10269 & 0.36 \\
\hline
\end{tabular}


Table 6 Results of displacement estimation: conventional method, method based on robust M-estimation and HLWE

\begin{tabular}{|c|c|c|c|c|c|c|}
\hline \multirow[t]{2}{*}{ Coordinate } & \multicolumn{4}{|c|}{ Displacements (mm) } & \multicolumn{2}{|c|}{$\hat{\sigma}_{H L W E}(\mathrm{~mm})$} \\
\hline & Simulated & Classical method & Robust M-estimation & HLWE & $\mathrm{MC}$ & $c=1.07$ \\
\hline $\mathrm{X} 1$ & 0.00 & 0.15 & 0.10 & 0.11 & 0.18 & 0.18 \\
\hline Y1 & 0.00 & 0.19 & 0.17 & 0.04 & 0.17 & 0.17 \\
\hline $\mathrm{X} 2$ & 0.00 & 0.03 & -0.09 & -0.15 & 0.11 & 0.11 \\
\hline Y2 & 0.00 & 0.09 & 0.01 & 0.06 & 0.13 & 0.13 \\
\hline $\mathrm{X} 3$ & -0.50 & -0.39 & -0.43 & $-0.56\left(-0.47^{*}\right)$ & 0.14 & 0.14 \\
\hline Y3 & -0.60 & -0.58 & -0.57 & $-0.50\left(-0.57^{*}\right)$ & 0.14 & 0.14 \\
\hline $\mathrm{X} 4$ & 0.00 & 0.11 & 0.10 & $0.29(0.00 *)$ & 0.30 & 0.29 \\
\hline Y4 & 0.00 & 0.13 & 0.06 & $0.45\left(0.08^{*}\right)$ & 0.24 & 0.26 \\
\hline X6 & 0.00 & 0.14 & 0.16 & 0.14 & 0.19 & 0.19 \\
\hline Y6 & 0.00 & -0.25 & -0.31 & -0.08 & 0.16 & 0.16 \\
\hline
\end{tabular}

* HLWE estimates computed when the point 3 or 4 were excluded from PRPs, respectively

not result from gross errors in the observation set, but from instability of one of PRPs (see, Duchnowski 2011). However, considering HLWEs of the coordinate changes and their estimated accuracy, one can conclude that the points 1,2 and 6 are stable. Thus, the outlying observations resulting from the instability of the point 3 do not influence the estimation results in a significant way. In such a context, we can say that HLWEs are robust against the outliers of that type (see also the next paragraph which gives some comments about robustness of the method). The results obtained for the unstable point 3 are also correct, namely they show that the point is actually unstable. The question might concern the point 4 , where the estimated displacements are rather big in relation to the respective estimates of the standard deviation; hence the point should be regarded as an unstable one. Herein, we know that it is stable but in general, we do not have such knowledge. Therefore, we should check if both point 3 and 4 are unstable or only one of them. This can be done by some additional analyses. Thus, let us repeat the computation in the case of the points 3 and 4 but this time let the point 3 be excluded from PRPs when stability of the point 4 is tested (and similarly, let us exclude the point 4 when the point 3 is checked). Note that we still have enough PRPs and the observations to perform such analysis. The respective HLWEs are presented in Table 6, they are marked by an asterisk. They confirm that the point 3 should be regarded as unstable, and the point 4 is actually stable. The example also shows that sometimes the process of testing the stability of PRPs should be two-staged when HLWEs (or HLEs) are applied.

The question is why the unstable point 3 influences the testing of stability of the other PRPs in different ways. The answer lies mostly in the configuration of the network. Note that the point 4 lays close to the unstable point 3 and relatively far from the stable points 1 , 2 or 6 . Another thing is the value of the breakdown point. Generally, the estimation gives satisfactory results if percentage of outlier is less than the value of the breakdown point. However, there are special cases when some robust estimates provide right solutions despite the fact that the percentage of outlying observations exceeds the breakdown point (see, $\mathrm{Xu}$ 2005). This is related to subjective break down points. Herein, this concerns the estimation of the displacements of the points 1, 2 and 6 . In such cases, up to $50 \%$ of the coordinate values are potentially outliers; however, the estimation results are still 
satisfactory. The problem of the subjective breakdown points is very interesting especially from the practical point of view. Its importance to HLWEs, however, requires further analysis which is beyond this paper.

The last two columns of Table 6 present the estimated standard deviations of HLWEs based on Monte Carlo simulations (performed separately for each coordinate) or computed from Eq. (5) by assuming that $c=1.07$ (such an approximate value is due to the values presented in Table 4). One can see that such estimates are almost always the same, which confirms the applicability of the simplified method of Eq. (5) in practice when one wants to avoid performing Monte Carlo simulations for each HLWE.

The analysis of stability of PRPs based on HLWEs leads to the same conclusions like other methods compared (they all find the point 3 unstable). The estimated displacements by the different methods are close to each other; however, they always differ from each other a little bit. Note that results obtained for HLWEs are based on a smaller number of the observations (we consider only a part of the whole network). The analysis performed here shows that HLWEs can be an alternative to the classical methods of deformation analysis; however, such an example cannot be regarded as a general comparison of the methods in question.

\section{Final conclusions}

The accuracy is a very important property of an estimate; in many particular practical applications it is just essential, for example, in deformation analysis. The law of propagation of variance is the most often method applied to assess such accuracy; however, such an approach is impossible for some estimation methods. In such a case, a good alternative is the method that is based on Monte Carlo simulations. The present paper shows such a method and its application in the case of chosen R-estimates, namely HLEs or HLWEs. The obtained results allow us to draw some interesting and important conclusions, which concern the general properties of the estimates in question as well as their practical applications.

Given the normal distribution of observations, one can say that the accuracy of HLEs (and respectively HLWEs) is comparable with the accuracy of LSEs; however, it is always a little bit worse (the differences between respective standard deviations are $\leq 5 \%$ as for HLEs, and $\leq 12 \%$ for HLWEs; however, considering practical applications of HLWEs in surveying or geodesy one can assume that such difference is $\leq 8 \%$ ). Note that the accuracy is not the only important property of R-estimates, namely such estimates are robust against outliers (Duchnowski 2011). Thus, HLEs (or HLWEs) seem to be an interesting and useful alternative for more conventional LSEs.

Considering distributions which kurtosis is different from zero, we can conclude that generally R-estimates are more accurate in the case of leptokurtic distributions and less accurate for platikurtic ones (with some interesting exceptions concerning the estimates of the expected value). Note that in many such cases HLEs (and also HLWEs) are more accurate than the respective LSEs. That concerns especially R-estimates of the shift.

The studies presented here also show that when number of observations increases then the accuracy of R-estimates generally becomes better in relation to the accuracy of respective LSEs. We can also conclude that for the lowest possible number of observations, namely when $n=2$ ( or $n=m=2$ ) the application of R-estimates seems unjustified. In the case of HLEs we would get exactly the same results as in LS method, and HLWEs have lower accuracy than LSEs. Note that for such small number of observations all R-estimates are not robust against outliers. 
It is also worth noting that all estimates discussed in the present paper belong to the same family; however, they may have different properties. That is clear when one compares results obtained for R-estimates of the expected value with the results obtained for R-estimates of the shift. The general impression and conclusions are similar in both cases; however, there are evident and important differences.

One of the aims of the paper was to propose a simplified method which can provide useful information about the accuracy of the chosen R-estimates. Given the formula in Eq. (5) and the values of the factor $c$ which are listed in Tables 1, 2, 3 and 4, someone who applies R-estimates can easily assess their accuracy. This was confirmed by the example presented in the present paper.

Open Access This article is distributed under the terms of the Creative Commons Attribution 4.0 International License (http://creativecommons.org/licenses/by/4.0/), which permits unrestricted use, distribution, and reproduction in any medium, provided you give appropriate credit to the original author(s) and the source, provide a link to the Creative Commons license, and indicate if changes were made.

\section{References}

Baarda W (1968) A testing procedure for use in geodetic networks. Netherlands Geodetic Commission Publications on Geodesy New Series vol 2, No. 5

Caspary WF (2000) Concepts of network and deformation analysis. University of New South Wales, Kensington

Cymerman M, Duchnowski R, Kopiejczyk A (2016) Selection of initial parameters in R-estimates applied to deformation analysis in leveling networks. J Surv Eng. doi:10.1061/(ASCE)SU.1943-5428.0000151

Duchnowski R (2008) R-estimation and its application to the LS adjustment. Boll Geod Sci Affini 67:17-32

Duchnowski R (2010) Median-based estimates and their application in controlling reference mark stability. J Surv Eng 136:47-52

Duchnowski R (2011) Robustness of the strategy for testing leveling mark stability based on rank tests. Surv Rev 43:687-699

Duchnowski R (2013) Hodges-Lehmann estimates in deformation analyses. J Geod 87(10):873-884

Duchnowski R, Wiśniewski Z (2014) Comparison of two unconventional methods of estimation applied to determine network point displacement. Surv Rev 46:401-405

Gurwitz C (1990) Weighted median algorithms for $\mathrm{L}_{1}$ approximation. BIT 30:301-310

Hekimoglu S, Erenoglu RC (2007) Effect of heteroscedasticity and heterogeneousness on outlier detection for geodetic networks. J Geod 81:137-148

Hettmansperger TP, McKean JW (2011) Robust nonparametric statistical methods. CRC Press, Boca Raton

Hodges JL Jr, Lehmann EL (1963) Estimates of location based on rank tests. Ann Math Stat 34:598-611

Kargoll B (2005) Comparison of some robust parameter estimation techniques for outlier analysis applied to simulated GOCE mission data. Gravity, geoid and space missions. In: IAG symposia, vol 129, pp 77-82. doi:10.1007/3-540-26932-0_14

Maritz JS, Jarrett RG (1978) A note on estimating the variance of the sample median. J Am Stat Assoc 73(361):194-196

Nowel K (2015) Robust M-estimation in analysis of control network deformations: classical and new method. J Surv Eng 141(4):1-10

Prószyński W (2015) Revisiting Baarda's concept of minimal detectable bias with regard to outlier identifiability. J Geod 89(10):993-1003

Reed JF III, Stark DB (1995) Monte Carlo generation of $\mathrm{S}_{\mathrm{B}}$ and $\mathrm{S}_{\mathrm{U}}$ distributions. Comput Methods Programs Biomed 47:157-165

Rousseeuw PJ, Verboven S (2002) Robust estimation in very small samples. Comput Stat Data Anal 40:741-758

Shi Y, Xu PL (2015) Comparing the estimates of the variance of unit weight in multiplicative error models. Acta Geod Geophys 50:353-363

Tadikamalla PR (1980) On simulating non-normal distributions. Psychometrika 45(2):273-279

Wiśniewski Z (1996) Estimation of the third and fourth order central moments of measurement errors from sums of powers of least squares adjustment residuals. J Geod 70(5):256-262 
Wiśniewski Z (2014) M-estimation with probabilistic models of geodetic observations. J Geod 88(10):941-957

Xu PL (2005) Sign-constrained robust least squares, subjective breakdown point and the effect of weights of observations on robustness. J Geod 79:146-159 